\title{
Cardiovascular safety of 'gliptin' therapy
}

Data from two trials designed to test the cardiovascular safety of the dipeptidyl peptidase 4 inhibitors saxagliptin and alogliptin, respectively, indicate that these drugs do not increase the incidence of ischaemic events. These findings were presented at the 2013 ESC Congress and simultaneously published in NEJM.

In the large SAVOR-TIMI 53 trial, investigators randomly allocated 16,492 patients with type 2 diabetes mellitus who had a history of, or were at risk of, cardiovascular events to saxagliptin or placebo, in addition to standard antihyperglycaemic therapy. During follow-up (median 2.1 years), the composite end point of cardiovascular death, myocardial infarction, or ischaemic stroke occurred in $7.3 \%$ and $7.2 \%$ of patients receiving saxagliptin or placebo, respectively (HR 1.00, $P<0.001$ for noninferiority). However, an increased rate of hospitalization for heart failure was reported in the saxagliptin group compared with those receiving placebo (3.5\% vs $2.8 \%$; HR $1.27, P=0.007$ ).
Researchers in the EXAMINE trial recruited 5,380 patients with type 2 diabetes and who had experienced an acute myocardial infarction or unstable angina requiring hospitalization in the preceding 15-90 days. Patients were randomly assigned to alogliptin or placebo, in addition to standard antihyperglycaemic and cardiovascular medications. During follow-up (median 18 months), death from cardiovascular causes, nonfatal myocardial infarction, or nonfatal stroke (the primary composite end point) occurred in $11.3 \%$ of patients receiving alogliptin and in $11.8 \%$ of those taking placebo (HR 0.96 , $P<0.001$ for noninferiority). The rate of hospitalization for heart failure was not included in the NEJM report, but William White, the lead researcher, states that "alogliptin was not associated with an increase in the composite end point of cardiovascular death and hospitalization for heart failure (7.4\% vs 7.5\% with placebo; HR 1.00, 95\% CI 0.82-1.21)".

Both inhibitors effectively lowered the blood glucose level-measurements of glycated haemoglobin were significantly reduced with either alogliptin or saxagliptin compared with placebo. In the EXAMINE trial, the incidence of hypoglycaemia was similar in the two groups; however, in the SAVOR-TIMI 53 trial, saxagliptin was associated with an increased rate of both major and minor hypoglycaemic events.

Taken together, the data from the SAVOR-TIMI 53 and EXAMINE trials suggest that gliptins have an acceptable cardiovascular safety profile. These drugs can provide effective glycaemic control, but might need to be combined with rigorous management of standard risk factors to reduce cardiovascular risk in patients with type 2 diabetes.

Gregory B. Lim

Original articles Scirica, B. M. et al. Saxagliptin and cardiovascular outcomes in patients with type 2 diabetes mellitus N. Engl. J. Med. doi:10.1056/NEJMoa1307684 | White, W. B. et al. Alogliptin after acute coronary syndrome in patients with type 2 diabetes. N. Engl. J. Med. doi:10.1056/NEJMoa1305889 